\title{
Correction to: Radius of Investigation in Pressure Transient Testing
}

\section{T. S. Ramakrishnan ${ }^{1}$ (D) - M. D. Prange ${ }^{1}$ - F. J. Kuchuk ${ }^{1}$}

Published online: 6 May 2020

(c) Springer Nature B.V. 2020

\section{Correction to: Transport in Porous Media}

\section{https://doi.org/10.1007/s11242-019-01367-y}

The arguments in Eqs. (16) and (18) have typographical errors in the original publication of the article. The corrected equations are provided below.

$$
\begin{aligned}
& \mathscr{P}\left(r_{d} \mid\left\{p_{m}\left(t_{i}\right), i=1, \ldots, N\right\}\right) \\
& \quad \propto \exp \left[-\frac{1}{2 \sigma^{2}} \sum_{i=1}^{N}\left\{p_{w}\left(t_{i} ; \bar{r}_{\mathrm{d}}\right)-p_{w}\left(t_{i} ; r_{d}\right)\right\}^{2}\right] \\
& \quad=\exp \left[-\frac{N}{2 \sigma^{2}}\left\langle\left\{p_{w}\left(t_{i} ; \bar{r}_{d}\right)-p_{w}\left(t_{i} ; r_{d}\right)\right\}^{2}\right\rangle\right] \\
& \quad \approx \exp \left[-\frac{1}{2 \sigma^{2} \Delta t} \int_{0}^{T}\left\{p_{w}\left(t ; \bar{r}_{d}\right)-p_{w}\left(t ; r_{d}\right)\right\}^{2} \mathrm{~d} t\right] .
\end{aligned}
$$

and

$$
\begin{aligned}
& \mathscr{P}\left(r_{d} \mid\left\{p_{m}\left(t_{i}\right), i=1, \ldots, N\right\}\right) \\
& \quad \propto \exp \left[-\frac{\left(r_{d}-\bar{r}_{d}\right)^{2} P^{2}}{2 \sigma^{2} \bar{r}_{d}^{2} \Delta t}\left(T \mathrm{e}^{-\frac{2 \bar{r}_{d}^{2}}{\eta T}}-\frac{2 \bar{r}_{d}^{2}}{\eta} \Gamma\left(0, \frac{2 \bar{r}_{d}^{2}}{\eta T}\right)\right)\right],
\end{aligned}
$$

A factor two in the denominator of an argument in Eqs. (28), (30), and (32) is missing. These equations should read

The original article can be found online at https://doi.org/10.1007/s11242-019-01367-y.

T. S. Ramakrishnan ramakrishnan@slb.com

M. D. Prange prange@alum.mit.edu

F. J. Kuchuk kuchuk1@slb.com

1 Schlumberger-Doll Research, 1 Hampshire St., Cambridge, MA 02139, USA 


$$
\begin{gathered}
p_{p}(t)=p_{0}+\frac{q \mu}{4 k r_{p}} \operatorname{erfc}\left(\frac{r_{p}}{2 \pi \sqrt{\eta t}}\right) . \\
p_{p}\left(t ; z_{b}\right)=p_{0}+P\left[\operatorname{erfc}\left(\frac{r_{p}}{2 \pi \sqrt{\eta t}}\right)+\frac{r_{p}}{2 \pi z_{b}} \operatorname{erfc}\left(\frac{z_{b}}{\sqrt{\eta t}}\right)\right] . \\
p_{p}^{(1)}\left(t ; r_{p}\right)=p_{0}+\operatorname{Perfc}\left(\frac{r_{p}}{2 \pi \sqrt{\eta t}}\right)+\frac{P r_{p}}{2 \pi \bar{z}_{b}} \operatorname{erfc}\left(\frac{\bar{z}_{b}}{\sqrt{\eta t}}\right) \\
-\frac{\operatorname{Pr}_{p}\left(z_{b}-\bar{z}_{b}\right)}{\pi \bar{z}_{b}}\left[\frac{\mathrm{e}^{-\frac{\bar{z}_{b}^{2}}{\eta t}}}{\sqrt{\pi \eta t}}+\frac{\operatorname{erfc}\left(\frac{\bar{z}_{b}}{\sqrt{\eta t}}\right)}{2 \bar{z}_{b}}\right] .
\end{gathered}
$$

\section{Reference}

Ramakrishnan, T.S., Prange, M.D., Kuchuk, F.J.: Radius of investigation in pressure transient testing. Trans. Porous Med. 131(3), 783-804 (2020)

Publisher's Note Springer Nature remains neutral with regard to jurisdictional claims in published maps and institutional affiliations. 